\title{
Be wall sources and migration in L-mode discharges after Be evaporation
}

\section{in the JET tokamak}

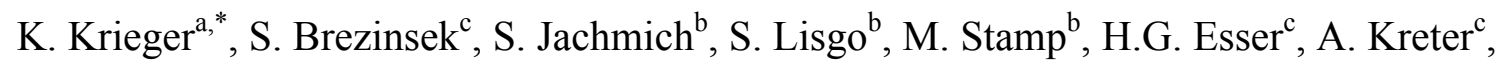 \\ S. Menmuir ${ }^{\mathrm{d}}$, Ph. Mertens ${ }^{\mathrm{c}}$, V. Philipps ${ }^{\mathrm{c}}$, P. Sundelin $^{\mathrm{d}}$ and JET EFDA contributors $^{+}$ \\ JET-EFDA, Culham Science Centre, OX14 3DB, Abingdon, UK \\ ${ }^{a}$ Max-Planck-Institut f. Plasmaphysik, Assoc. EURATOM-IPP, Garching, Germany \\ ${ }^{b}$ Euratom/UKAEA Fusion Assoc., Culham Science Centre, Oxon OX14 3DB, UK \\ ${ }^{c}$ Inst. f. Energieforsch., FZJ, EURATOM Assoc., Trilateral Euregio Cluster, Jülich, Germany \\ ${ }^{d}$ Association EURATOM - VR, Fusion Plasma Physics, EES, KTH, Stockholm, Sweden
}

${ }^{+}$See Appendix of M.L.Watkins et al., Proc. 21st Int. Conf. Chengdu, 2006, IAEA (2006).

\begin{abstract}
First wall material erosion and migration after fresh Be evaporation in the JET tokamak were studied in a series of consecutive identical L-mode discharges. The evolution of Be and C wall and divertor sources towards steady state conditions after deposition of a finite amount of Be at the carbon first wall of JET by the beryllium evaporation procedure provides a data set for benchmarking impurity transport simulations. Furthermore the experiment serves as a reference case for comparison of Be erosion to that of the planned ITER-like wall experiment with mainly Be plasma facing components (PFCs) in the main chamber. The experimental results confirm the migration pattern obtained by campaign integrated accounting of impurity sources and sinks, which is characterised by the main chamber wall and outer divertor as erosion dominated zones and migration of eroded material predominantly to the inner divertor where the material is finally deposited at the target plates.
\end{abstract}


PACS: 28.52.Fa, 52.25.Vy, 52.40.Hf, 52.65.Pp

JNM keywords: B0100, I0100, P0500, R0900, T0100

PSI-18 keywords: Beryllium, DIVIMP, Erosion \& Deposition, Impurity Sources, Impurity Transport

*Corresponding author address: MPI für Plasmaphysik, Boltzmannstr. 2, Garching, Germany

*Corresponding author email: krieger@ipp.mpg.de

Presenting author: Karl Krieger

\section{Introduction}

Beryllium will be used as main chamber armour material for ITER. Migration and redeposition of eroded beryllium is considered a potential hazard because Be might contribute to formation of tritium inventories by co-deposition and because of possible deterioration of tungsten armour by alloy formation with Be. In previous studies at JET, campaign integrated $\mathrm{Be}$ and $\mathrm{C}$ sources at the main chamber wall were compared to post campaign deposition measurements at retrieved divertor tiles [1-3]. It turned out that both materials are predominantly deposited in the inner divertor $[4,5]$. This observation can be interpreted as a consequence of erosion dominating at main chamber wall and outer divertor strike point area and migration of eroded material to the inner divertor following the experimentally observed plasma boundary flow pattern from the low-field side to the high-field side of the boundary plasma [6-8]. To study the time scales of the corresponding erosion and migration processes in more detail and, like in trace impurity injection experiments, to obtain data suitable for benchmarking of impurity transport modelling, dedicated experiments with focus on a particular discharge scenario are required. Although ITER relevant plasma scenarios all rely on H-mode confinement, L-mode discharges are particularly useful as base scenario for transport studies because they allow avoiding the additional complication of ELMs for both experimental diagnostics and modelling. 


\section{Diagnostic setup}

Local $\mathrm{Be}$ and $\mathrm{C}$ sources were measured spectroscopically in the visible spectral range. The evolution of BeII , $\mathrm{CII}$ and $\mathrm{D}_{\beta}$ line intensities from discharge to discharge allows to derive estimates for the relative change of beryllium and carbon surface concentration and erosion rates respectively. Figure 1 shows a subset of the available lines of sight of the JET KS3A spectrometer viewing at the inner (I) and outer (O) divertor and horizontally at the mid-plane main chamber wall $(\mathrm{H})$. Viewing chord $\mathrm{H}$ at the plasma mid-plane was additionally coupled to a high resolution overview spectrometer in cross-dispersion arrangement [9] for separate determination of inboard and outboard wall sources by Zeeman analysis [10]. Charge exchange spectrometry [11] was used to monitor the evolution of the carbon plasma concentration. Furthermore, the material deposition rate at the inner divertor close to the pump duct area was detected by a quartz microbalance (QMB) [12].

\section{Discharge scenario}

A series of 15 identical L-mode discharges (2.5MA, 2.4T) with $1.5 \mathrm{MW}$ neutral beam heating was performed with fresh Be wall coverage provided by Be evaporation in the plasma main chamber previous to the session. The wall status before Be evaporation was documented by a reference discharge with identical configuration at the end of the preceding session. To avoid excessive local erosion in the main chamber, the magnetic configuration (Figure 1) was tailored to ensure sufficient wall clearance and the discharge schedule was programmed without limiter start-up phase and minimised plasma-limiter interaction during the plasma start-up and ramp-down phase with early X-point formation already in the ramp-up phase. A low triangularity configuration $(\delta=0.2)$ with both strike points located symmetrically at the horizontal target plates was used to optimise the position of the divertor impurity sources with respect to the available spectroscopic viewing chords and QMB diagnostics. Each discharge 
had $\approx 10$ s flat top time with $\approx 7$ seconds neutral beam heating and stationary plasma wall clearance $(10 \mathrm{~cm}$ at low field side, $14 \mathrm{~cm}$ at high field side) and strike-point positions.

The Be evaporation with 4 toroidally separated evaporator heads was actually planned with extended time $(4 \mathrm{~h})$ and at elevated evaporator temperature $\left(1000^{\circ} \mathrm{C}\right)$, which would have resulted in evaporation of $40 \mathrm{~g}$ Be. With the accidentally used standard parameters of 2 hours Be evaporation at $850^{\circ} \mathrm{C}$ evaporator temperature the evaporated mass of Be was only $0.2 \mathrm{~g}$. Assuming even distribution of the evaporated $\mathrm{Be}$ in the main chamber, the corresponding layer thickness was therefore limited to $\approx 0.6 \mathrm{~nm}$. In addition, the evaporator in vessel octant 1 closest to the spectroscopy diagnostics operated at reduced performance $\left(700^{\circ} \mathrm{C}\right)$ due to power supply problems. It turned out, however, that despite these problems, the Be coverage was sufficient to produce data of good quality.

\section{Beryllium and carbon sources}

With constant plasma flux and plasma temperature local BeII $(527.1 \mathrm{~nm})$ and CII $(589.1 \mathrm{~nm})$ line intensities normalised to the intensity of the $\mathrm{D}_{\beta}$ Balmer line - a measure for the local deuterium recycling flux - provide a suitable measure for the respective surface fraction. Figure 2 shows the evolution of the BeII/ $\mathrm{D}_{\beta}$ flux ratio during the discharge series for the midplane main chamber wall $(\mathrm{H})$ and for the outer $(\mathrm{O})$ and inner (I) divertor viewing chords. The data points represent signal average of one second during the neutral beam heated plasma phase. Both at the main chamber wall and the outer divertor region one observes a factor 6-8 increase relative to the pre-Be evaporation level decreasing by $\approx 30 \%(\mathrm{H})$ and $\approx 50 \%(\mathrm{O})$ in the first 3 discharges and subsequently followed by a slower continuous decrease over the remaining 12 discharges of the experiment. In contrast, the BeII/D $\mathrm{D}_{\beta}$ flux ratio from the inner divertor increased only by a factor 3 over the level before Be evaporation and also showed a significantly lower decrease rate than the main chamber and outer divertor signals. This can be understood if one takes into account that Be layers were deposited at the inner target plates 
already in many discharges before this experiment leading to a smaller relative change of $\mathrm{Be}$ sources compared to erosion dominated areas where the initial left-over Be coverage was much smaller.

On the carbon sources the effect of the Be evaporation is anti-correlated to the beryllium behaviour. Figure 3 shows, as in Figure 2 for beryllium, the evolution of the $C I I / D_{\beta}$ flux ratio. At the main chamber wall, the CII signal drops by $\approx 50 \%$ after Be evaporation and reaches steady state after the first two discharges. A similar behaviour is observed in the divertor, however with a smaller drop of $\approx 25 \%$. Correspondingly, the deposition rate at the inner divertor louvres measured by QMB, which is dominated by hydrocarbon molecules, remained, after a slight initial increase in the first 4 discharges, constant during the experiment. These results show that the preceding Be evaporation procedure did not result in complete Be wall coverage, at least at the wall of the vessel segment with the spectroscopy diagnostics. This is expected because of the suboptimal performance of the adjacent $\mathrm{Be}$ evaporator (section 3). The resulting toroidal asymmetry has to be analysed in detail by numerical tracing of the evaporated Be to the plasma facing wall.

For the main chamber there is finally an ambiguity in the position of the impurity source. The corresponding line emission might originate from either or both the high field side wall or low field side wall. Analysis of Zeeman line splitting for the mid-plane viewing chord $(\mathrm{H})$ allowed to resolve both fractions. The results show approximately equal source fractions at inboard and outboard wall for both carbon and beryllium, however with limited accuracy because the plasma temperature at both emission locations had to be included in the data analysis as free parameters.

\section{Carbon plasma concentration}

Spectroscopic erosion flux measurements can only provide data for a few selected vessel locations. A more global quantity is the plasma concentration of a given impurity, which is, 
for a given scenario with similar plasma parameters directly correlated to the product of erosion flux and screening factor averaged over the entire wall area. Because of diagnostic failure, Be CXRS lines were partially cut off and only the carbon concentration, $f_{C}$, could be derived from the corresponding line emission. Figure 4 shows the evolution of $f_{C}$ at two radial positions. As in Figure 2 and Figure 3, the data points represent averages over a one second time window. The drop of the carbon concentration after Be evaporation and the following increase rate are similar to the corresponding evolution of the local CII source rates. In 3 discharges, the plasma was also shifted by $6 \mathrm{~cm}$ towards the central wall. This leads to increased erosion of $\mathrm{Be}$ and $\mathrm{C}$ at the central column (Figure 2 and Figure 3), however, at the same time the carbon concentration decreases significantly. From these observations one can infer that carbon sources at the outboard limiters contribute to a greater extent to the carbon flux into the plasma than $\mathrm{C}$ sources at the central column.

\section{Transport modelling}

The results presented in sections 4 and 5 can be interpreted in a qualitative way. A detailed picture of the evolving wall sources and quantitative derivation of the impurity source flux from the measured photon intensities requires the use of numerical transport modelling. A problem in all available codes is caused by a topological restriction of computational grids, which are derived from magnetic geometry, to allow only direct plasma-wall contact at two independent poloidal grid boundaries, usually at the inner and outer target plates. This limitation results generally in large gaps between the main chamber walls and the grid (Figure 5a). This leads to large uncertainties in the modelling of main chamber erosion and redeposition distributions. The impurity transport code DIVIMP was extended to allow handling of extended grids where the gaps between wall and original grid are closed by additional cells [13] (Figure 5b). First simulations of Be migration were done for both grids to study the influence of the grid-wall gaps. The plasma background was computed using the 
two-point onion skin solver OEDGE/SOL28 [13] included in DIVIMP with boundary conditions provided by upstream and target profiles of electron density and temperature. For simplicity, only erosion by direct plasma contact in the divertor was assumed. Figure $6 \mathrm{a}$ and $\mathrm{b}$ show the resulting distribution of Be deposition along the vessel wall for standard (a) and extended grid (b) respectively. For the standard grid, deposition at the wall was computed by attributing deposition of a particle leaving the grid to the nearest wall element outside the grid. Although the fraction of particles eroded in the divertor reaching the main chamber wall is approximately the same ( $6 \%$ for standard grid vs. $7.3 \%$ for the extended grid), the distribution of the deposition shows significant differences. With the now available extended grid topology, one has a tool for greatly improved analysis of impurity sources and migration, in particular for the plasma main chamber.

\section{Conclusions}

Post-campaign analysis of Be coverage on retrieved divertor tiles showed Be deposition mainly at the inner divertor. This observation, combined with similar results for carbon, shows that redeposition prevails over gross erosion in the inner divertor while at the outer target plate Be deposited after Be evaporation is completely re-eroded. Consequently, one would expect a smaller impact of Be evaporation on the Be source in the inner divertor compared to erosion dominated wall regions. This is qualitatively confirmed by the results of the current experiment, where the Be sources at both the main chamber wall and the outer divertor show much stronger variations than in the inner divertor. Impurity transport simulations have been prepared with improved modeling of the plasma-wall contact in the main chamber. This provides an essential tool both for quantitative interpretation of the new results and for benchmarking the transport simulations to improve the quality of extrapolation for ITER and other planned fusion experiments. 


\section{Acknowledgements}

"This work, supported by the European Communities under the contract of Association between EURATOM/IPP-Garching, was carried out within the framework of the European Fusion Development Agreement. The views and opinions expressed herein do not necessarily reflect those of the European Commission." 


\section{References}

[1] G.F. Matthews, M. Stamp, P. Coad, et al., Material Migration in JET, 30th EPS Conference on Contr. Fusion and Plasma Phys., St. Petersburg, Europhysics Conference Abstracts 27A, P-3.198 (2003).

[2] R.A. Pitts, J.P. Coad, D.P. Coster, et al., Plasma Physics And Controlled Fusion 47 (2005) B303.

[3] G.F. Matthews, Journal Of Nuclear Materials 337-39 (2005) 1.

[4] J. Likonen, E. Vainonen-Ahlgren, J.P. Coad, et al., Journal Of Nuclear Materials 33739 (2005) 60.

[5] J.P. Coad, P. Andrew, S.K. Erents, et al., Journal Of Nuclear Materials 363 (2007) 287.

[6] R.A. Pitts, J. Horacek, W. Fundamenski, et al., Journal Of Nuclear Materials 363 (2007) 505.

[7] S.K. Erents, R.A. Pitts, W. Fundamenski, et al., Plasma Physics And Controlled Fusion 46 (2004) 1757.

[8] N. Asakura, S. Sakurai, K. Itami, et al., Journal Of Nuclear Materials 313 (2003) 820.

[9] S. Brezinsek, A. Pospieszczyk, M.F. Stamp, et al., Journal Of Nuclear Materials 337339 (2005) 1058.

[10] P. Pugno, A. Kallenbach, D. Bolshukhin, et al., Journal Of Nuclear Materials 290 (2001) 308 .

[11] C.R. Negus, C. Giroud, A.G. Meigs, et al., Review of Scientific Instruments 77 (2006)

[12] H.G. Esser, G. Neill, P. Coad, et al., Fusion Engineering and Design 66-8 (2003) 855.

[13] S. Lisgo, P. Borner, C. Boswell, et al., Journal Of Nuclear Materials 337-39 (2005) 139. 


\section{Figure captions}

Figure 1: Poloidal cross-section of JET with observation positions of visible range spectrometers at main chamber wall and divertor target plates. Flux surfaces in the plot correspond to the flat-top phase of the L-mode discharges.

Figure 2: Evolution of BeII intensity normalised to $\mathrm{D}_{\beta}$ for main chamber $(\mathrm{H})$, outer $(\mathrm{O})$ and inner divertor (I). The hatched area denotes the effect of a $6 \mathrm{~cm}$ plasma shift towards the central wall.

Figure 3: Evolution of CII intensity normalised to $\mathrm{D}_{\beta}$ for main chamber $(\mathrm{H})$, outer $(\mathrm{O})$ and inner divertor (I). The hatched area denotes the effect of a $6 \mathrm{~cm}$ plasma shift towards the central wall.

Figure 4: Evolution of carbon plasma concentration $\mathrm{f}_{\mathrm{C}}=\mathrm{n}_{\mathrm{C}} / \mathrm{n}_{\mathrm{e}}$ in plasma centre (normalised radius $\rho=0.08)$ and outer plasma region $(\rho=0.82)$. The hatched areas denote the effect of a 6 cm plasma shift towards the central wall.

Figure 5: Computational grids derived from magnetic configuration of discharge \#68124 for simulation of Be and $\mathrm{C}$ transport. a) standard grid configuration. b) extended grid configuration without gaps to main chamber wall.

Figure 6: Be wall deposition for a simulation with Be source limited to the divertor target plates, a) for standard grid configuration, b) for extended grid configuration. 
Figure 1

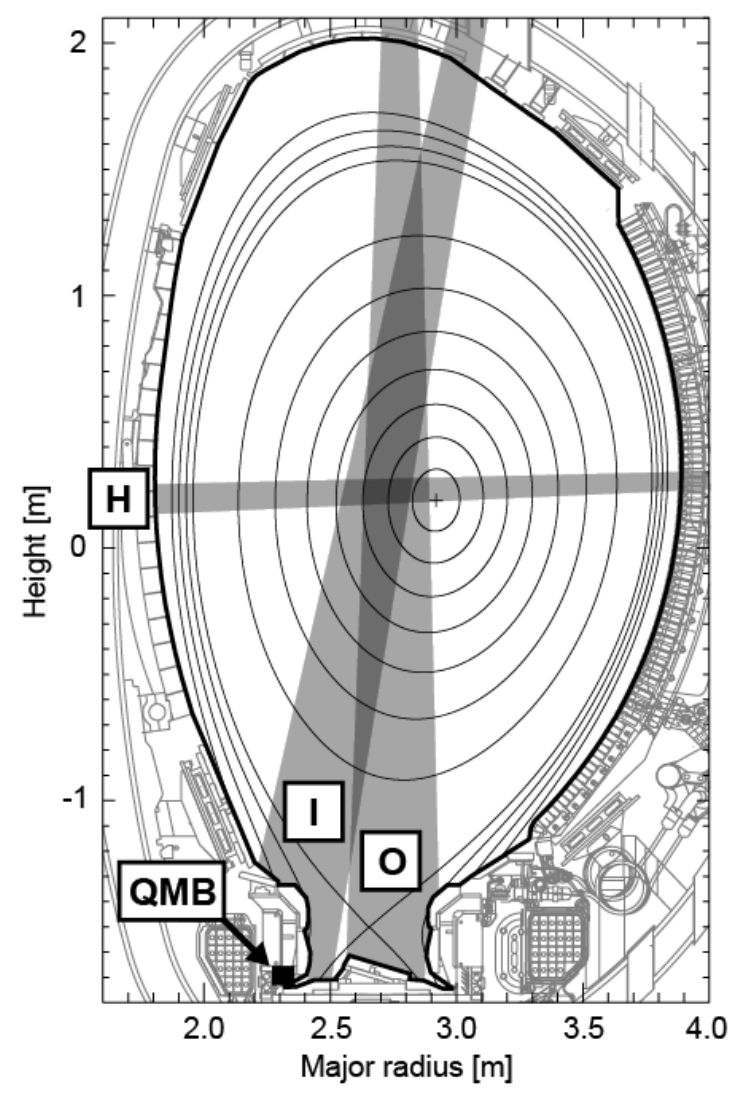


Figure 2
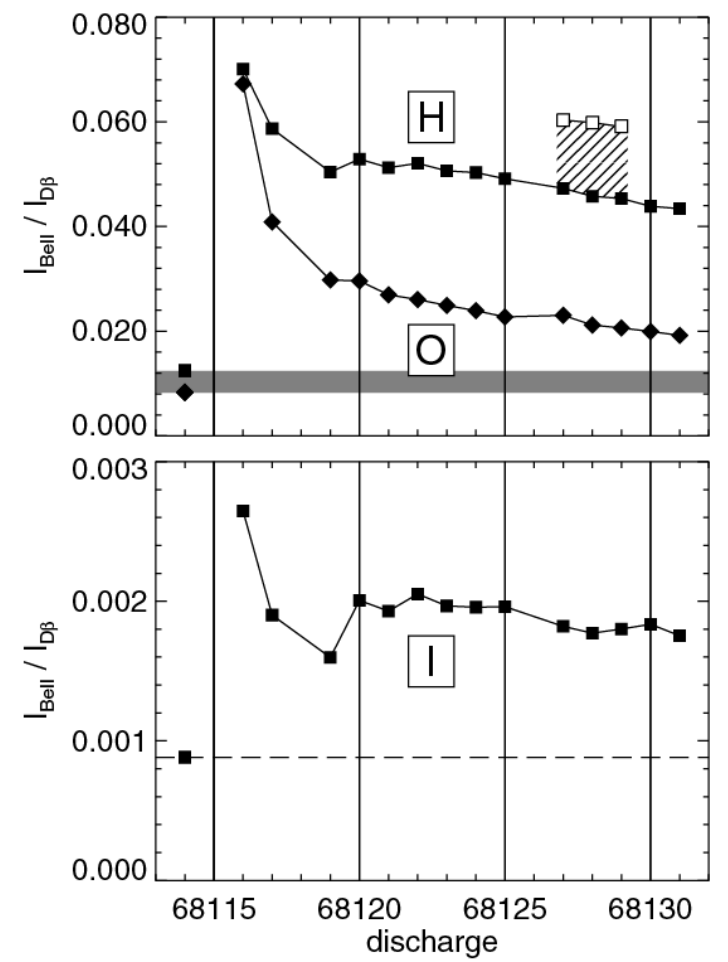
Figure 3
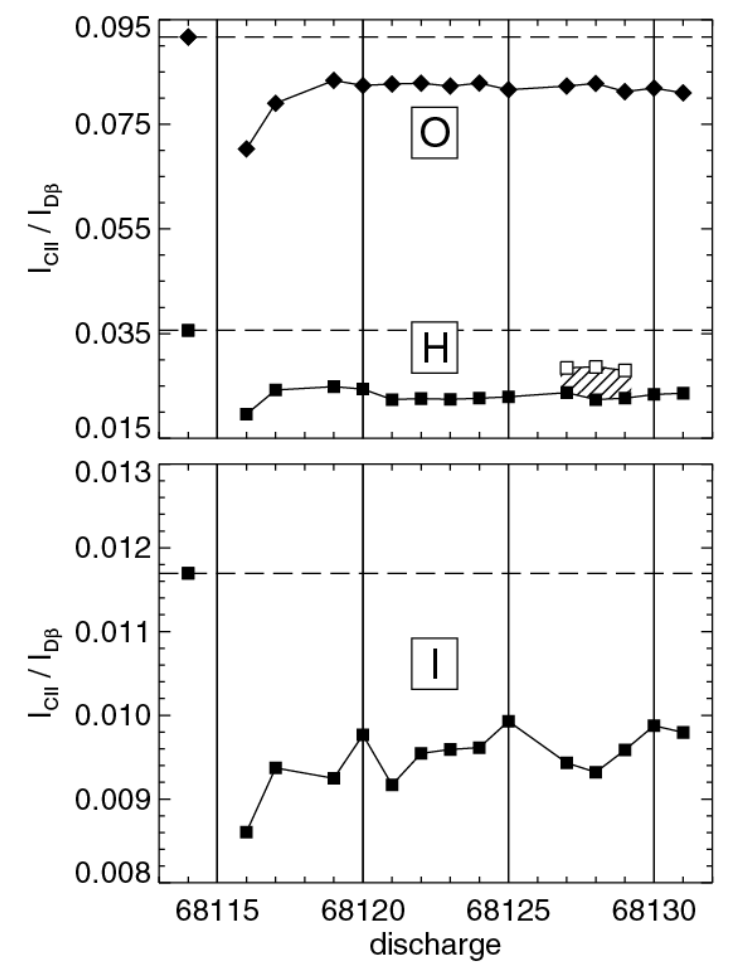
Figure 4

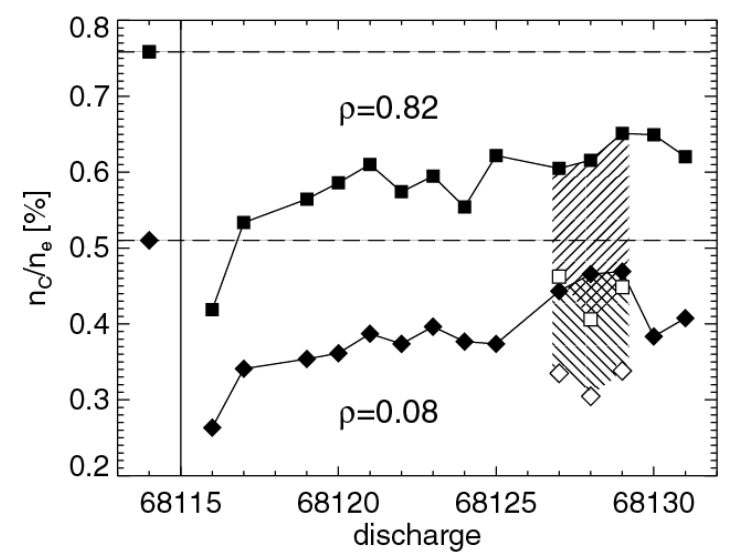


Figure 5

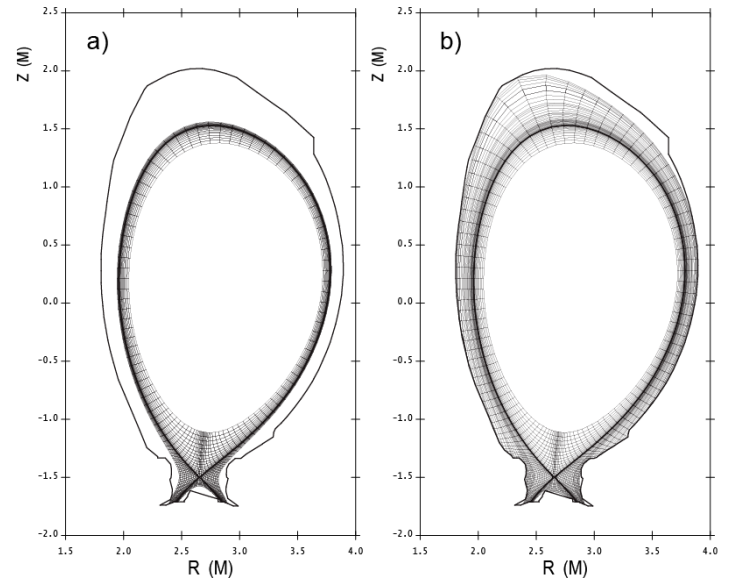


Figure 6
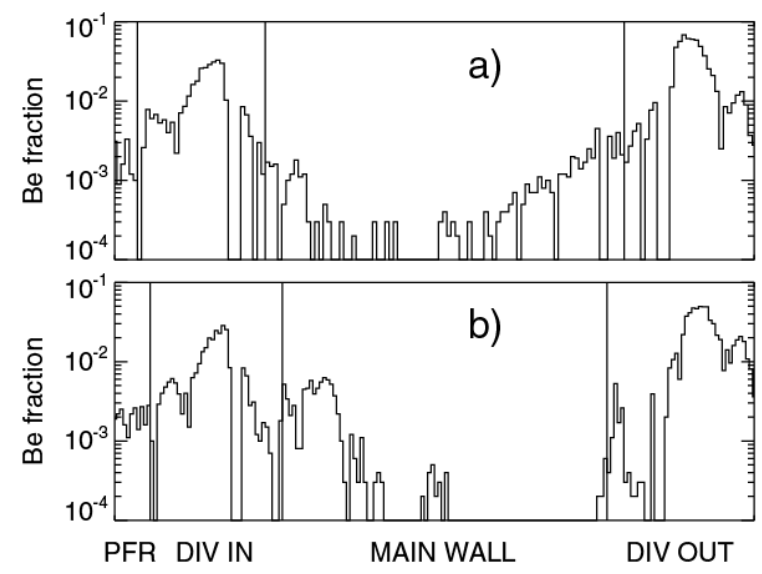